Published in final edited form as:

J Am Geriatr Soc. 2017 October ; 65(10): 2158-2160. doi:10.1111/jgs.14942.

\title{
Network for Investigation of Delirium across the U.S. (NIDUS): Advancing the Field of Delirium with a New Interdisciplinary Research Network
}

\author{
Donna M. Fick, PhD, RN ${ }^{1}$, Andrew D. Auerbach, $\mathrm{MD}^{2}$, Michael S. Avidan, $\mathrm{MBBCh}^{3}$, Jan \\ Busby-Whitehead, MD ${ }^{4}$, E. Wesley Ely, MD, MPH ${ }^{5,6,7}$, Richard N. Jones, ScD ${ }^{8}$, Edward R. \\ Marcantonio, MD, SM ${ }^{9,10}$, Dale M. Needham, MD, PhD ${ }^{11}$, Pratik Pandharipande, MD $^{7,12}$, \\ Thomas N. Robinson, MD ${ }^{13}$, Eva M. Schmitt, PhD ${ }^{14}$, Thomas G. Travison, PhD ${ }^{10,14}$, and \\ Sharon K. Inouye, MD, MPH ${ }^{9,10,14}$ for the NIDUS Delirium Network \\ ${ }^{1}$ College of Nursing, College of Medicine, Department of Psychiatry, The Pennsylvania State \\ University, University Park, PA, USA \\ ${ }^{2}$ Division of Hospital Medicine, University of California San Francisco, San Francisco, CA, USA \\ ${ }^{3}$ Department of Anesthesiology, Washington University School of Medicine, St. Louis, Missouri, \\ USA
}

${ }^{4}$ Division of Geriatric Medicine/Center for Aging and Health, Department of Medicine, University of North Carolina School of Medicine, Chapel Hill, NC, USA

${ }^{5}$ Geriatric Research, Education, and Clinical Center, Department of Veterans Affairs Medical Center, Tennessee Valley Health Care Center, Nashville, TN, USA

${ }^{6}$ Division of Allergy, Pulmonary and Critical Care Medicine, Vanderbilt University Medical Center, Nashville, TN, USA

${ }^{7}$ Center for Health Services Research, Vanderbilt University Medical Center, Nashville, TN, USA

${ }^{8}$ Departments of Psychiatry and Human Behavior and Neurology, Brown University Warren Alpert Medical School, Providence, RI, USA

${ }^{9}$ Department of Medicine, Beth Israel Deaconess Medical Center, Boston, MA, USA

${ }^{10}$ Harvard Medical School, Boston, MA, USA

${ }^{11}$ Department of Medicine, Division of Pulmonary and Critical Care Medicine, Johns Hopkins University School of Medicine, Baltimore, MD, USA

Corresponding Author: Donna M Fick, PhD, College of Nursing, 201 Nursing Sciences Building, University Park, PA 16802 (dmf21@ psu.edu). Contact phone (814) 865-9325.

Conflict of Interest: The authors have no conflicts of interest related to this manuscript and have completed COI disclosure forms.

Author Contributions: DMF participated in the study design and drafted the initial manuscript. SKI conceived the study design and conceptualization, and critical revision of the manuscript. All authors have contributed to the manuscript preparation and final revisions.

Sponsor's Role: The NIDUS Network is supported by the National Institute on Aging, Grant No.R24AG054259 (SKI). The funding agencies had no role and the authors retained full autonomy in the preparation of this manuscript. 
${ }^{12}$ Division of Anesthesiology Critical Care Medicine, Department of Anesthesiology, Vanderbilt University Medical Center, Nashville, TN, USA

${ }^{13}$ Department of Surgery, University of Colorado, School of Medicine, Aurora, CO, USA

${ }^{14}$ Institute for Aging Research, Hebrew SeniorLife, Boston, MA, USA

Delirium, a manifestation of brain organ dysfunction, presents acutely with impaired attention and disorganized thinking, and imposes a high degree of caregiver burden. Delirium can occur at any age, but occurs more often in older adults due to their increased vulnerability, affecting over 2.6 million in the U.S. each year ( $>5$ persons every minute). Typically following acute illness, hospitalization, or surgery, delirium often leads to loss of independence, cognitive decline, and increased morbidity and mortality. Moreover, delirium is associated with excess healthcare costs of over $\$ 164$ billion per year. ${ }^{1,2}$ Delirium can be difficult to detect and manage, and is receiving increased attention in the medical literature. ${ }^{3-5}$

A number of factors, including cognitive impairment, infection, dehydration and psychoactive medications are predictive of delirium (Inouye et al., 2014). In most cases, delirium is multi-factorial, which makes finding effective approaches for prevention and treatment highly challenging, and research studies methodologically complex. Importantly, delirium can be prevented with a multi-dimensional clinical strategy. ${ }^{6}$ Despite its importance, delirium is not well understood and is vastly under-recognized. Fundamental advances in diagnosis and treatment of delirium are lagging. The pathophysiology of delirium remains poorly understood and there are no biomarkers to serve as diagnostic indicators or therapeutic targets. ${ }^{7}$ Although the number of measures for screening and diagnosis of delirium has increased in the past decade, the proportion of patients with unrecognized delirium remains high. ${ }^{8,9}$

In November 2016, a team of delirium experts led by Sharon K. Inouye, M.D., M.P.H., Professor of Medicine at Harvard Medical School and Director of the Aging Brain Center at Hebrew SeniorLife's Institute for Aging Research (IFAR), were awarded a \$3.7 million grant over five years. This award is the first of its kind from the National Institute on Aging (NIA)/ National Institutes of Health, and is intended to build an interdisciplinary collaborative network of delirium researchers nationally and internationally. Dr. Inouye, along with 12 interdisciplinary experts in delirium research, will create the Network for Investigation of Delirium across the U.S. (NIDUS) and beyond to foster collaboration and accelerate scientific discovery in delirium. NIDUS will unite delirium researchers from 27 organizations across the U.S. in focused collaborative efforts. NIDUS takes an interdisciplinary approach that includes many medical specialties, nursing, social work, rehabilitation, cognitive science, neuroscience, epidemiology, biostatistics, and related fields, and will create a collaborative network to bring new approaches to accelerate scientific discovery in delirium.

NIDUS is comprised of two research cores and three task forces. The Research Resources and Database Core, led by Edward Marcantonio, MD, SM, Pratik Pandharipande, MD, and Thomas Travison, $\mathrm{PhD}$, will develop a database of ongoing delirium studies to encourage 
collaboration and secondary analyses of existing data. The Measurement and Harmonization Core, led by Richard Jones, $\mathrm{ScD}$ and Dale Needham, MD, PhD will develop resources to help researchers choose tools for assessing delirium and delirium severity. The three NIDUS task forces, Pilots and Innovation, Mentorship and Career Development, and Dissemination are further described in Table 1.

The NIDUS network provides structured activities for interdisciplinary delirium researchers, including scientific symposia and delirium research Boot Camps. NIDUS is offering pilot grant opportunities (Table 1), and providing information and resources on delirium, which can be accessed at: https://deliriumnetwork.org/. The kick-off for presenting NIDUS to professionals will occur at the American Delirium Society (ADS) meeting in Nashville, TN on June 6, 2017. This will be an opportunity to meet and talk with NIDUS investigators, and to learn first-hand about our exciting opportunities, resources, and activities through the cores and task forces. The ADS meeting is June 4-6, 2017 and registration information is available at: https://americandeliriumsociety.org/ads-2017.

Another key activity of NIDUS is the annual delirium research "Boot Camp" where researchers with a passion for delirium research can apply for the 2.5-day intensive training session in delirium research. The Boot Camp is intended for delirium researchers across disciplines-either newly entering the field or changing research directions-- who have previous training in the fundamentals of clinical research, with the goal of enhancing the cadre of trained scientists focusing on delirium. Accepted applicants will be expected to submit a specific research proposal, and will be paired with a NIDUS mentor. The study of delirium is complex and methodologically challenging, and the Boot Camp provides approaches to help address some of these complexities. The Boot Camp will be held November 1-3, 2017 in Boston, MA, and will include interactive training in delirium assessment, mock grant review, individual methodological consultations, and didactics on the latest updates, biomarkers, neuroimaging, electrophysiology, and mixed methods in delirium research. Travel grants are available to selected applicants. The application can be accessed on the NIDUS website at https://deliriumnetwork.org/.

We have reached a pivotal moment where the importance of delirium is gaining traction among scientists, clinicians and the general public. Approximately one year ago the iDelirium group and website (www.idelirium.org) were established. iDelirium is a federation of international delirium organizations advocating for delirium with a unified voice, which launched the inaugural World Delirium Day on March 15, 2017. While there is growing awareness of its importance, many large gaps remain in our scientific understanding of delirium and in the implementation of best practices into clinical care. While not directly affiliated, the NIDUS scientific network complements the work of iDelirium by helping address the many gaps and challenges in delirium science, establishing a research infrastructure and databases to increase collaboration among delirium researchers globally, and helping to mentor the next generation of interdisciplinary delirium scientists. The need for additional research on delirium is compelling and urgent. Clinicians and researchers across fields are encouraged to join the broad range of interdisciplinary experts in delirium research as leaders, scientists and innovators. Together, we are working to advance the field 
of delirium, with the ultimate goal of improving clinical care for this common and devastating condition.

\section{Acknowledgments}

This work is dedicated to the memory of Jane Ann McDowell for her lifelong work in delirium.

\section{References}

1. Leslie DL, Inouye SK. The importance of delirium: Economic and societal costs. J Am Geriatr Soc. 2011; 59:S241-S243. DOI: 10.1111/j.1532-5415.2011.03671.x [PubMed: 22091567]

2. Inouye SK, Westendorp RG, Saczynski JS. Delirium in elderly people. Lancet. 2014; 383:911-922. DOI: 10.1016/S0140-6736(13)60688-1 [PubMed: 23992774]

3. Steis MR, Fick DM. Are nurses recognizing delirium? A systematic review. J Gerontol Nurs. 2008; 34:40-48. DOI: 10.3928/00989134-20080901-12

4. Wilber ST, Ondrejka JE. Altered mental status and delirium. Emerg Med Clin North Am. 2016; 34:649-665. DOI: 10.1016/j.emc.2016.04.012 [PubMed: 27475019]

5. Lee A, Mu JL, Joynt GM, et al. Risk prediction models for delirium in the intensive care unit after cardiac surgery: a systematic review and independent external validation. Br J Anaesth. 2017; 118:391-399. DOI: 10.1093/bja/aew476 [PubMed: 28186224]

6. Hshieh TT, Yue J, Oh E, et al. Effectiveness of multicomponent nonpharmacological delirium interventions. A meta-analysis. JAMA Intern Med. 2015; 175:512-520. DOI: 10.1001/ jamainternmed.2014.7779 [PubMed: 25643002]

7. Davis DH, Muniz-Terrera G, Keage HA, et al. Association of delirium with cognitive decline in late life: A neuropathologic study of 3 population-based cohort studies. JAMA Psychiatry. 2017; 74:244-251. DOI: 10.1001/jamapsychiatry.2016.3423 [PubMed: 28114436]

8. McCoy TH Jr, Snapper L, Stern TA, Perlis RH. Underreporting of delirium in statewide claims data: Implications for clinical care and predictive modeling. Psychosomatics. 2016; 57:480-488. DOI: 10.1016/j.psym.2016.06.001 [PubMed: 27480944]

9. Morandi A, Davis D, Bellelli G, et al. The diagnosis of delirium superimposed on dementia: An emerging challenge. J Am Med Dir Assoc. 2017; 18:12-18. DOI: 10.1016/j.jamda.2016.07.014 [PubMed: 27650668] 


\section{Table 1}

NIDUS Cores and Task Forces

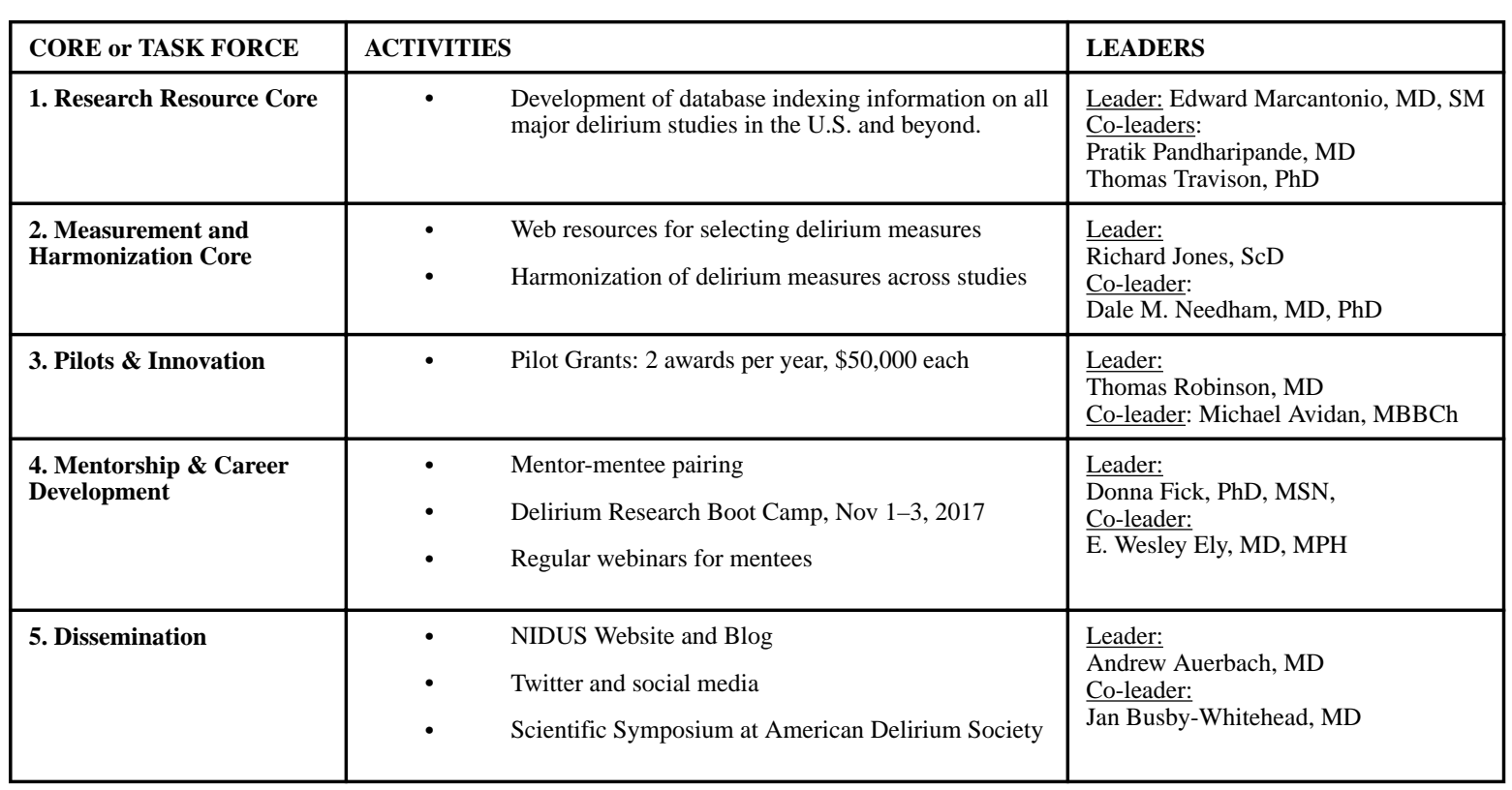

